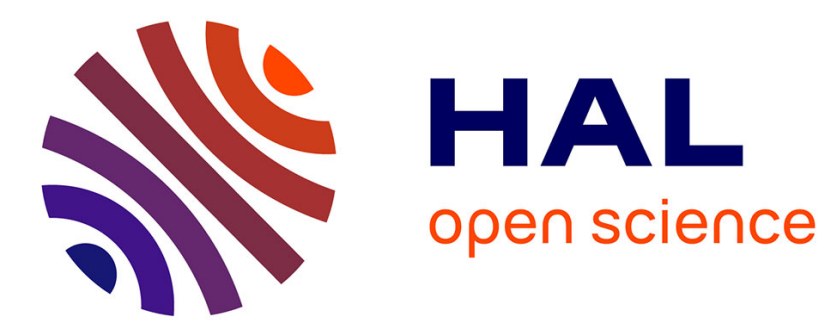

\title{
Estimation of the Free Core Nutation parameters from SG data: sensitivity study and comparative analysis using linearized Least-Squares and Bayesian methods
}

Séverine Rosat, Nicolas Florsch, Jacques Hinderer, Muriel Llubes

\section{- To cite this version:}

Séverine Rosat, Nicolas Florsch, Jacques Hinderer, Muriel Llubes. Estimation of the Free Core Nutation parameters from SG data: sensitivity study and comparative analysis using linearized Least-Squares and Bayesian methods. Journal of Geodynamics, 2009, 48 (3-5), pp.331. 10.1016/j.jog.2009.09.027 . hal-00594412

\section{HAL Id: hal-00594412 \\ https://hal.science/hal-00594412}

Submitted on 20 May 2011

HAL is a multi-disciplinary open access archive for the deposit and dissemination of scientific research documents, whether they are published or not. The documents may come from teaching and research institutions in France or abroad, or from public or private research centers.
L'archive ouverte pluridisciplinaire $\mathbf{H A L}$, est destinée au dépôt et à la diffusion de documents scientifiques de niveau recherche, publiés ou non, émanant des établissements d'enseignement et de recherche français ou étrangers, des laboratoires publics ou privés. 


\section{Accepted Manuscript}

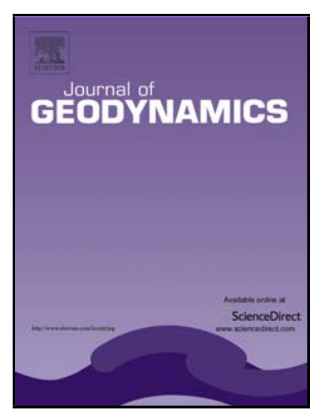

Title: Estimation of the Free Core Nutation parameters from SG data: sensitivity study and comparative analysis using linearized Least-Squares and Bayesian methods

Authors: Séverine Rosat, Nicolas Florsch, Jacques Hinderer, Muriel Llubes

PII: S0264-3707(09)00094-5

DOI:

Reference: doi:10.1016/j.jog.2009.09.027

To appear in: $\quad$ Journal of Geodynamics

Please cite this article as: Rosat, S., Florsch, N., Hinderer, J., Llubes, M., Estimation of the Free Core Nutation parameters from SG data: sensitivity study and comparative analysis using linearized Least-Squares and Bayesian methods, Journal of Geodynamics (2008), doi:10.1016/j.jog.2009.09.027

This is a PDF file of an unedited manuscript that has been accepted for publication. As a service to our customers we are providing this early version of the manuscript. The manuscript will undergo copyediting, typesetting, and review of the resulting proof before it is published in its final form. Please note that during the production process errors may be discovered which could affect the content, and all legal disclaimers that apply to the journal pertain. 
1 Estimation of the Free Core Nutation parameters from SG data: sensitivity study and

4 Séverine Rosat $^{(1),{ }^{*}}$, Nicolas Florsch $^{(2)}$ Jacques Hinderer $^{(1)}$ and Muriel Llubes ${ }^{(3)}$

5 (1) Institut de Physique du Globe de Strasbourg (UMR 7516 CNRS, Université de 6 Strasbourg/EOST), 5 rue René Descartes, 67084 Strasbourg Cedex, France comparative analysis using linearized Least-Squares and Bayesian methods

(2) UPMC, Campus JUSSIEU, 4, place Jussieu, 75252 PARIS Cedex 05; UMMISCO/IRD, France, and Dpt of Mathematics and Applied Mathematics, UCT, South Africa.

(3) Université de Toulouse, OMP - LEGOS, 14 Av. Edouard Belin, F-31400 Toulouse, France

* Corresponding author: Severine.Rosat@eost.u-strasbg.fr Fax: (+33) (0)3 90240291

\begin{abstract}
The Free Core Nutation (FCN) is investigated with the help of its resonance effect on the tidal amplitudes in Superconducting Gravimeter (SG) records of the GGP network. The FCN resonance parameters are combined in a resonance equation involving the Earth's interior parameters. The sensitivity of the FCN parameters to the diurnal tidal waves demonstrates that the quality factor of the FCN is strongly dependent on the accuracy of the imaginary part estimates of the gravimetric factors close to the resonance. The weak amplitude of $\Psi_{1}$ tidal wave on the Earth, which is the closest in frequency to the FCN, in addition to errors in ocean loading correction, explains the poor determination of the quality factor $Q$ from surface gravimetric data. The inversion of tidal gravimetric factors leads to estimates of the period, $Q$ and resonance strength of the FCN. We show that, by inverting $\log (Q)$ instead of $Q$, the results using the least-squares method optimized using the Levenberg-Marquardt algorithm are in agreement with the Bayesian probabilistic results and agree with the results obtained
\end{abstract}


1 from VLBI nutation data. Finally, a combined inversion of 7 GGP European SG data is

2 performed giving $\mathrm{T}=428+/-3$ days and $7762<\mathrm{Q}<31989$ (90\% C.I.). An experimental

3 estimate of the internal pressure Love number is also proposed.

4

5 Keywords: Free Core Nutation, Superconducting Gravimeter, Bayesian analysis

\section{Introduction}

8

Because of the fluidity of the core, the Earth has a rotational mode, called the free core nutation (FCN) with a period almost diurnal in Earth-fixed coordinates. The FCN parameters (period, damping) strongly depend on the coupling mechanism at the core-mantle boundary (flattening, topography, electro-magnetic coupling...). The FCN can be detected by its effect on the Earth's rotation, using the VLBI network analyses, or by studying its effects on the gravity field. As the tidal potential contains some diurnal components, a resonance occurs in the diurnal frequency band. This resonance effect can be observed in time-varying gravity data continuously recorded on the Earth's surface by Superconducting Gravimeters (SGs) of the Global Geodynamics Project (GGP) network (Crossley et al., 1999). The FCN resonance in gravity data is commonly represented by a damped harmonic oscillator model that we invert in order to determine the FCN frequency, quality factor $Q$ and the transfer function of the mantle (or the resonance strength). The usual approach to solve this non-linear inverse problem is to use a linearized least-squares method optimized based on the LevenbergMarquardt algorithm (Marquardt, 1963 - Numerical Recipes Fortran Chapter 15.5- see for instance Defraigne et al. 1994 and 1995; Sato et al., 2004; Ducarme et al., 2007). However Florsch and Hinderer (2000) have demonstrated the inadequacy of using such a least-squares method, because the statistical distribution of $Q$ is definitely not Gaussian. They have 
1 proposed instead the use of a Bayesian approach to invert the FCN parameters, since the

2 Bayesian method better propagates the information to the parameters.

Neuberg et al. (1987) first proposed an inversion of stacked gravity tide measurements in central Europe to retrieve the FCN parameters using the Marquardt optimized linearized least-squares. Then Defraigne et al. (1994) extended the gravity stack to the nutation observations. In those past studies, the obtained $Q$-value was abnormally small and sometimes even negative. Sato et al. (2004) used $1 / Q$ instead of $Q$ as a parameter to be inverted using a modified Marquardt least-squares method since $1 / Q$ seems to be Gaussian (Sato et al., 1994). 
In the first part we describe the FCN resonance model. Then, we review the theory of the Bayesian method and the Levenberg-Marquardt optimization algorithm applied to linearized least-squares. A qualitative study is then performed to check the sensitivity of the gravity factors to the FCN parameters. Finally, we invert the FCN resonance parameters using a combination of 7 European SG time-series.

\section{The FCN resonance model}

The basic equation used to describe the resonance of the FCN in the tidal gravity is usually written as (Hinderer et al., 1991a):

$$
\widetilde{\delta}_{j}=\widetilde{\delta}_{r e f}+\frac{\widetilde{a}}{\sigma_{j}-\widetilde{\sigma}_{n d}}
$$

where $\widetilde{\delta}_{j}$ corresponds to the complex gravimetric factor observed for every tidal wave of frequency $\sigma_{j}, \widetilde{\sigma}_{n d}=\sigma_{n d}^{R}+i \sigma_{n d}^{I}$ is the complex eigenfrequency of the FCN, $\widetilde{a}=a^{R}+i a^{I}$ refers to the resonance strength corresponding to the response of the whole Earth to the FCN. The quantity $\widetilde{\delta}_{r e f}$ is the value of the gravimetric factor without any resonance process (classical tidal gravimetric factor); it is also the asymptotic value of $\widetilde{\delta}_{\mathrm{j}}$ for frequencies far away from the resonance frequency. The eigenperiod $T$ of the FCN expressed in sidereal days in the rotating frame is related to $\sigma_{n d}^{R}$ by:

$$
T=\frac{2 \pi}{\sigma_{n d}^{R}},
$$

where $\sigma_{n d}^{R}$ is expressed in radian per sidereal day. In the inertial reference frame, the period can be written:

$$
T^{\prime}=\frac{1}{\sigma_{n d}^{R} C-1}
$$


1 where $\mathrm{C}=\frac{86164}{15 \times 86400}$ and $\sigma_{n d}^{R}$ is given in degrees/solar hour. The quality factor $Q$,

2 expressing the damping due to all the physical processes involved in the resonance, is defined

3 as $Q=\frac{\sigma_{n d}^{R}}{2 \sigma_{n d}^{I}}$. The quantities $\sigma_{n d}^{R}$ and $\sigma_{n d}^{I}$ are positive by definition, therefore they should

4 follow a log-normal distribution law (Tarantola, 2005; Florsch and Hinderer, 2000) to avoid

5 possible negative values. It is therefore recommended to include the a priori positivity of $Q$ in

6 the model by changing the variable $Q=10^{x}$ and inverting for $x$, instead of $Q$.

$7 \quad$ Florsch and Hinderer (2000) performed the inversion by treating $\widetilde{\delta}_{r e f}$ as an unknown

8 and showed that a correlation exists between the real parts $\delta_{r e f}^{R}$ and $\sigma_{n d}^{R}$, and between $a^{R}$

9 and $\delta_{r e f}^{R}$, but the correlation between $a^{R}$ and $T$ is much stronger. As $\widetilde{\delta}_{r e f}$ has a weak influence

10 on the values of $T$ and $Q$, we do not include this parameter in the inversion process. In

11 previous studies (e.g. Defraigne et al. 1994 - 1995; Ducarme et al., 2007), the observed value

12 for the tidal wave $\mathrm{O}_{1}$ was used as the reference gravimetric factor. In our case we will use the

13 mean value of the theoretical inelastic amplitude factors of the $\mathrm{O}_{1}$ and $\mathrm{OO}_{1}$ waves computed

14 for the Wahr-Dehant model (Wahr, 1974; Dehant, 1987). By doing so, we suppose that the

15 scale factors of the SG used here are accurate enough, which is usually true (better than $0.3 \%$

16 accuracy, e.g. Amalvict et al., 2001; Sato et al., 2004). We could also have normalized by the

17 observed O1 amplitude as done by Sato et al. (2004) but we suppose that the scaling error is

18 negligible with respect to the ocean loading uncertainty.

19 The equations relative to the resonance model are written: 
1

$$
\left\{\begin{array}{l}
\delta_{j}^{R}=\delta_{r e f}+\frac{a^{R}\left(\sigma_{j}-\sigma_{n d}^{R}\right)-a^{I} \frac{\sigma_{n d}^{R} 10^{-x}}{2}}{\left(\sigma_{j}-\sigma_{n d}^{R}\right)^{2}+\left(\frac{\sigma_{n d}^{R} 10^{-x}}{2}\right)^{2}} \\
\delta_{j}^{I}=\frac{\frac{a^{R} \sigma_{n d}^{R} 10^{-x}}{2}+a^{I}\left(\sigma_{j}-\sigma_{n d}^{R}\right)}{\left(\sigma_{j}-\sigma_{n d}^{R}\right)^{2}+\left(\frac{\sigma_{n d}^{R} 10^{-x}}{2}\right)^{2}} \text { with } \delta_{r e f}=\frac{\delta_{O 1}^{R}+\delta_{O O 1}^{R}}{2} .
\end{array}\right.
$$

2 Equations (2) will be our formulation for the resonance model.

Hinderer (1986), Hinderer and Legros (1989), Defraigne (1992) and Legros et al. (1993) have written the analytical expression for the resonance strength as a function of the

5 Earth's interior parameters:

6

$$
\widetilde{a}=-\widetilde{\delta}_{2} \frac{A}{A_{m}} e\left(\sigma\left(1-\frac{A}{A_{f}} \frac{\gamma}{e}\right)-\frac{A_{m}}{A} \widetilde{\sigma}_{n d}^{\prime}\right)-\widetilde{\delta}_{1} \frac{A}{A_{m}} e \Omega\left(1-\frac{\gamma}{e}\right)
$$

7 where $\gamma$ is a compliance (Mathews et al., 2002) related to the geodetic parameter $q_{0}$ (ratio of centrifugal acceleration to gravitational acceleration) and the Love number $h^{f}$ by

$9 \gamma=\frac{q_{0}}{2} h^{f} . \widetilde{\delta}_{1}$ is the pressure Love number that represents the gravity response to the pressure potential acting at the CMB (Hinderer et al. 1991a). $e$ is the dynamic ellipticity of the Earth,

$11 \Omega$ its rotation rate and $A / A_{m}$ is the ratio of Earth to mantle moments of inertia.

$12 \widetilde{\sigma}_{n d}^{\prime}=-\Omega \frac{A}{A_{m}}\left(e^{f}-\beta\right)$ is the FCN frequency in the inertial reference frame $\left(\widetilde{\sigma}_{n d}^{\prime}=\widetilde{\sigma}_{n d}+\Omega\right)$,

$13 e^{f}$ is the dynamical flattening of the CMB and $\beta$ the compliance defined by $\beta=\frac{q_{0}}{2} h_{1}^{f} \cdot h_{1}^{f}$ is the displacement Love number at the CMB associated with the fluid pressure on the mantle caused by the relative rotation. 
Legros et al. (1993) also considered the effect of the inner core including the Free Inner Core Nutation (FICN) resonance effect. We neglect it here as the effect would be too small to be seen in SG records.

In the following, we will estimate the period $T$, the quality factor $Q$, as well as the real and imaginary parts of the resonance strength $\widetilde{a}$ using two methods: a probabilistic approach based on the Bayesian inversion and the more classical linearized least-squares optimized using the Levenberg-Marquardt algorithm.

\section{A review of the methods}

a. The Bayesian approach

The Bayesian inversion consists in propagating the information (or knowledge) provided by the measurements through an assumed physical model (perfectly or probabilistically known) to the parameters and to include the a priori knowledge of the model parameters. Both the data and the model parameters are described with probability distributions. The Bayesian approach preserves the full knowledge provided by the data combined with the physical law and the a priori information on the data and model parameters. Therefore, it is the most suitable method to perform the inversion of non-linear problems (Tarantola and Valette, 1982a, b). For more details about the Bayesian method, please refer to Florsch and Hinderer (2000).

The Bayesian probability distribution of the parameter vector $\theta$ is given by:

$$
p(\boldsymbol{\theta})=p\left(x, \sigma_{n d}^{R}, a^{R}, a^{I}\right)=k \exp \left\{-\frac{1}{2} \sum_{j}\left[\left(\frac{\operatorname{Re}\left(\delta_{j}^{t h}\right)-\operatorname{Re}\left(\delta_{j}\right)}{\Delta \operatorname{Re}\left(\delta_{j}\right)}\right)^{2}+\left(\frac{\operatorname{Im}\left(\delta_{j}^{t h}\right)-\operatorname{Im}\left(\delta_{j}\right)}{\Delta \operatorname{Im}\left(\delta_{j}\right)}\right)^{2}\right]\right\},
$$

where $k$ is a normalisation factor in order that the integral of this equation is unity, $R e$ denotes the real part and $I m$ the imaginary part, $\delta_{j}$ holds for the $j^{\text {th }}$ measurement value of the 
1 gravimetric factor, th refers to the theoretical value, and $\Delta \delta$ is the error on $\delta$ (standard 2 deviation).

The previous formula gives the general probability laws for the parameter vector $\theta$. In order to obtain the law for one or two parameters, we compute the marginal pdfs by integration of the probability function over selected parameters. For instance, the joint pdf integrated with respect to $\sigma_{n d}^{R}$ is defined by:

$$
p_{x, a^{R}, a^{I}}\left(x, a^{R}, a^{I}\right)=\int p\left(x, \sigma_{n d}^{R}, a^{\mathrm{R}}, a^{I}\right) d \sigma_{n d}^{R} .
$$

Notice that when integrating over selected parameters, we limit a priori the space parameter on a finite domain. Two further integrations of the pdf lead to the marginal probability law for each of the parameters.

\section{$\underline{\text { b. The linearized least-squares approach }}$}

As for the Bayesian method, the linearized least-squares approach is a subset of the non-linear least squares generalized by Tarantola and Valette (1982b). The only difference is how to treat a priori information, and what pdfs are involved. The least-squares method is based on the determination of the best-fit parameters by minimizing the merit function:

$$
\chi^{2}=\sum_{j} \frac{\left|\widetilde{\delta}_{j}-\left(\delta_{r e f}+\frac{\widetilde{a}}{\sigma_{j}-\widetilde{\sigma}_{n d}}\right)\right|^{2}}{\left(\Delta \widetilde{\delta}_{j}\right)^{2}}
$$

However, when the model is non-linear, the minimization must proceed iteratively given initial values for the parameters. The procedure finishes when $\chi^{2}$ stops decreasing. The $\chi^{2}$ function is linearized to a quadratic form depending on the Hessian matrix for the inverseHessian method or the gradient of $\chi^{2}$ for the steepest descent method. The LevenbergMarquardt (also called Marquardt method; Marquardt, 1963) algorithm is in fact a smooth variant between these two methods. The Marquardt method works very well in practice and 
1 has become a standard of non-linear least-squares routines. It has been widely used in

2 previous FCN retrieval studies like in Neuberg et al. (1987), Richter and Zürn (1986), Zürn

3 and Rydelek (1991), and Defraigne et al. (1994, 1995). We refer to the Numerical Recipes

4 (Press et al., 1992) Chapter 15.5 for the optimization subroutines.

5 The set of linear equations $\sum_{j} \alpha_{k j} \Delta \theta_{j}=\beta_{k}$ is solved for the increments $\Delta \theta_{j}$ that, added to the 6 current trial parameters, give the next approximation. The matrix $\alpha$ is called the curvature

7 matrix and is equal to one-half times the Hessian matrix: $\alpha_{k j}=\frac{1}{2} \frac{\partial^{2} \chi^{2}}{\partial a_{k} \partial a_{j}}$ and $\beta_{k}=-\frac{1}{2} \frac{\partial \chi^{2}}{\partial a_{k}}$,

where $a_{k}$ (or $a_{j}$ ) corresponds to one parameter. In the Marquardt optimization algorithm, the increments are related to the curvature matrix through a "damping factor" $\lambda$ as $\Delta \theta_{j}=\frac{1}{\lambda \alpha_{j j}} \beta_{j}$. When $\lambda$ is very large, the diagonal of the curvature matrix is dominant and the solution tends to the initial parameters (the increments tend toward zero). On the other hand, as $\lambda$ approaches zero, the increments become very large and we may have a divergence problem. The damping factor $\lambda$ is also known as the Marquardt factor. The damping factor effectively constrains the range of values that the increments $\Delta \theta_{j}$ can take.

The model used for the inversion is described by equation (2). In order to impose the positivity of the quality factor $Q$, we perform the change of variable $x=\log _{10}(Q)$, as for the Bayesian approach, then we minimize equation (5). We also estimate the damping factor $\lambda$, which best minimizes $\chi^{2}$.

\section{Sensitivity and correlations between the FCN parameters}

In order to check the sensitivity of the diurnal tidal wave amplitudes to the FCN parameters, we perform some qualitative studies of the resonance transfer function presented in equation (2) and based on the previously estimated values. We have $\sigma_{n d}^{R} \cong 7.30910^{-5} \mathrm{rad} / \mathrm{s}$, 
$1 Q \approx 210^{4}$ (based on VLBI results, e.g. Mathews et al., 2002), $a^{R} \approx 3.410^{-9} \mathrm{rad} / \mathrm{s} \approx 710^{-4}$

$2 \mathrm{deg} / \mathrm{h}$ and $a^{I} \approx 210^{-10} \mathrm{rad} / \mathrm{s} \approx 410^{-5} \mathrm{deg} / \mathrm{h}$ (e.g. Hinderer et al., 1989; Florsch and Hinderer,

3 2000; see next section).

4 From equation (2) and with these values, for any diurnal waves of angular frequency $\sigma_{j}$, we

5 can see that the quantity $a^{I} \frac{\sigma_{n d}^{R}}{2 Q}$ is negligible with respect to

$6 a^{R}\left(\sigma_{j}-\sigma_{n d}^{R}\right)$ and $\left(\frac{\sigma_{n d}^{R}}{2 Q}\right)^{2}<<\left(\sigma_{j}-\sigma_{n d}^{R}\right)^{2}$, hence the amplitude gravimetric factor can be

7 approximated by:

8

$$
\left\{\begin{array}{l}
\delta_{j}^{R} \approx \delta_{r e f}+\frac{a^{R}}{\left(\sigma_{j}-\sigma_{n d}^{R}\right)} \\
\delta_{j}^{I} \approx \frac{\frac{a^{R} \sigma_{n d}^{R} 10^{-x}}{2}+a^{I}\left(\sigma_{j}-\sigma_{n d}^{R}\right)}{\left(\sigma_{j}-\sigma_{n d}^{R}\right)^{2}}
\end{array}\right.
$$

9 For diurnal waves situated far from the resonance, i.e. for $\left(\sigma_{j}-\sigma_{n d}^{R}\right)>>3.10^{-8} \mathrm{rad} / \mathrm{s}$, we

10 have $\delta_{j}^{I} \approx \frac{a^{I}}{\left(\sigma_{j}-\sigma_{n d}^{R}\right)}$. Therefore, the FCN quality factor is only constrained by the imaginary

11 part of the gravimetric factor of the diurnal waves close to the resonance $\left(K_{l}, \Psi_{l}\right.$ and $\left.\Phi_{l}\right)$ and

12 from equation (6) we can see that the imaginary part of the resonance strength is mainly

13 constrained by the imaginary part of the gravimetric factors. However, the imaginary parts of

14 the tidal gravity factors are poorly determined, especially for $\Psi_{1}$ and $\Phi_{1}$ that have small

15 amplitudes (particularly a small imaginary part) and are therefore very sensitive to the ocean

16 loading correction error (Sato et al., 2004). We have tested the influence of the error of the

17 imaginary part of the gravimetric factors on the resulting pdfs of the Bayesian inversion. For that, we have considered synthetic gravimetric factors computed from equation (2) with $x=4$, 
$1 T=430$ days, $a^{R}=610^{-4} \mathrm{deg} / \mathrm{h}$ and $a^{I}=-510^{-5} \mathrm{deg} / \mathrm{h}$. Then we assume an error of $0.1 \%$ on

2 the real part of the gravimetric factors for nine diurnal waves (from $Q_{1}$ to $O O_{1}$ waves). We

3 increase the relative error on the imaginary part from $1 \%$ to $100 \%$, and we also test the case

4 when the error on $\delta^{\mathrm{I}}\left(\Psi_{l}\right)$ and $\delta^{\mathrm{I}}\left(\Phi_{l}\right)$ becomes larger than their values themselves (relative

5 uncertainty larger than 100\%). The corresponding pdfs are plotted in Fig. 1. When the relative

6 error on the imaginary part of $\Phi_{1}$ and $\Psi_{1}$ reaches $100 \%$, the pdf for $x$ begins to be non-

7 Gaussian and tends to an asymmetric probability law (Fig. 1 (b)). If we still increase the

8 relative error on $\delta^{\mathrm{I}}\left(\Psi_{l}\right)$ and $\delta^{\mathrm{I}}\left(\Phi_{l}\right)$ to $200 \%$ (Fig. 1 (c)) or increase the error for all the waves

9 to $100 \%$ (Fig. 1 (e)), then the probability law for $x$ is definitely not Gaussian any more and tends to the infinity towards larger values. For a relative error of $50 \%$, the pdf for $x$ can still be approximated by a Gaussian law (Fig. 1 (d)). In a previous paper, Florsch and Hinderer (2000) obtained a probability law for $x$ that tends to infinity: that is because of the large errors they had on the imaginary parts of the smallest diurnal waves $\left(\Psi_{l}\right.$ and $\left.\Phi_{l}\right)$. As a consequence, if the estimations of the gravimetric factors were more accurate (i.e. with relative errors smaller than $50 \%$ ), then the probability law for $x$ should be Gaussian. Moreover, using VLBI nutation data, for which the amplitudes of the equivalent $\Phi_{1}$ and $\Psi_{1}$ nutations are high and well-determined, Rosat and Lambert (2009) have indeed obtained a Gaussian-law for $x$. 
1 determination of the gravimetric factors at the site. The results of the least-squares inversion

2 lead to the conclusion that, with an error of 0.004 on the real part of $\widetilde{\delta}_{\psi_{1}}$ the a posteriori error on $T$ from the least-squares inversion is about 2.3 days. For $x=\log _{10}(Q)$, the correlation with $\delta_{\Psi_{1}}^{I}$ is much stronger and $x$ varies between 4.2 and 4.6, corresponding to $Q$-values between 15849 and 39811, when $\delta_{\Psi_{1}}^{I}$ ranges from 0.002 to 0.012 . Therefore the uncertainty on the estimation of $Q$ is large even if the ocean loading correction was perfect. The error on $Q$ is therefore mostly due to the fitting error of $\Psi_{l}$ because of its small amplitude. There is also a strong correlation between $\delta_{\Psi_{1}}^{I}$ and $a^{I}$. Similar computations could be done for $K_{l}$ and $\Phi_{l}$.

We have shown that the diurnal waves close to the resonance are very sensitive to the FCN parameters. Besides, some correlations exist between the FCN resonance parameters. As shown by the tilted shapes in Fig. 1, two strong correlations exist between $a^{R}$ and $T$, on the one hand, and between $a^{I}$ and $x$, on the other hand. Physically it comes from the response of the whole Earth to the FCN that depends also on the complex eigenfrequency of the core oscillation. Indeed, the damping of the nearly-diurnal free wobble introduced through the complex eigenfrequency could be due to the dissipation by viscomagnetic and topographic coupling at the CMB and to the anelasticity (Mathews et al. 2002) while the imaginary part introduced in the resonance strength, reflects the anelasticity of the Earth which is related to the imaginary part of the Love numbers. As a consequence, the $Q$-factor is physically correlated to the imaginary part of the resonance strength through the anelasticity of the interface.

Florsch and Hinderer (2000) have also shown that the reference gravimetric factor is correlated to $a^{R}$ and hence to the real part of $\widetilde{\delta}_{1}$. As we have an uncertainty (usually less than $0.3 \%$ ) on the SG scale factor, this error will be propagated to the estimation of $\widetilde{\delta}_{1}$. As we will 
1 see in the next part, this scaling error of $0.3 \%$ is smaller than the uncertainty that we obtain

2 for $\widetilde{\delta}_{1}$.

3 The exploration performed by Florsch and Hinderer (2000) has shown that the

4 imaginary part of the strength $a^{I}$ is close to zero with an error much larger than the value

5 itself. They proposed to cancel this parameter in order to restrict the scanning to a 3-parameter

6 space. However, $a^{I}$ is strongly correlated to $Q$ particularly for $Q$-values less than 1000: putting

$7 a^{I}=0$ forces $Q$ to be around 2600 (cf. Fig. 2 (a)). Note that in this case (elastic model), the

8 obtained $T$ value is close to 465 days, which is the value inferred by Sasao et al. (1980) for an

9 elastic Earth with a hydrostatic CMB flattening.

When imposing $a^{I}$ equal to a value close to the estimation from the 4-D inversion, i.e. $a^{I}=-5$

$10^{-5} \mathrm{deg} / \mathrm{h}$, then we obtain a most probable values for $Q$ of $21241+/-6956$ (cf. Fig. 2 (b))

which is in agreement with predictions of the MHB model (Mathews et al., 2002) and with VLBI nutation observations (e.g. Lambert and Dehant, 2007; Koot et al., 2008). In the next part, we will finally estimate the FCN resonance parameters.

\section{Combined GGP inversion}

Here we propose to analyze, in the spectral domain, 7 tidal European SG observations to invert the FCN parameters. Contrary to Ducarme et al. (2009), we do not compute averaged gravimetric factors but combine them by using relation (4) and we suppose that the resonance strength $\widetilde{a}$ is the same for all the sites. Because $\widetilde{a}$ corresponds to the mantle response to the 
1 FCN, supposing a homogeneous response at all SG sites enables to retrieve the global

2 parameters (cf. paragraph 2) like the internal pressure Love number at the CMB. The weight

3 in equation 4 is the combination of the standard deviation of the fitted gravimetric factor by

4 ETERNA software and of the ocean loading error.

The SG sites considered here are Bad-Hambourg (H1, Germany), Moxa (M1, Germany), Membach (MB, Belgium), Medicina (MC, Italy), Strasbourg (ST, France), Vienna (VI, Austria) and Wettzell (W1, Germany). H1 (resp. M1, W1) refer to the lower sphere of the double-sphere SG installed at these German sites. The record length that has been used to retrieve the gravimetric factors from the ETERNA tidal analysis is larger than 5 years for every time-series. These sites have been chosen because they are relatively far from the ocean (except Membach) and their SGs are known to be well calibrated (better than $0.3 \%$ accuracy). The raw data have been corrected for gaps, spikes, steps and other disturbances so that a tidal analysis with ETERNA software (Wenzel, 1996) is possible. Before the tidal analysis, the minute data are decimated to $1 \mathrm{~h}$ (using a filter with a cut-off period of $3 \mathrm{~h}$ ). The ETERNA software then performs a least-squares fit to tides, local air pressure and instrumental drift to retrieve the complex gravimetric factors, the residual gravity, an adjusted barometric admittance, and a polynomial drift function.

The data to be inverted are the complex gravimetric factors corrected for the ocean tide loading effect according to FES2004 ocean model (Lyard et al. 2006, for ocean loading computation see for instance Llubes et al. 2008). We have attributed a nominal error of 0.02 $\mathrm{nm} / \mathrm{s}^{2}$ on the ocean loading correction vector. This value has been roughly estimated based on the comparison between different ocean tide models. The inversion is carried out for the four parameters $\left(x, \sigma_{n d}^{R}, a^{R}, a^{I}\right)$ on a $101^{4}$ points space. The explored parameter space is defined based on the results by Florsch and Hinderer (2000), namely $x$ spans from 3 to 8, $T$ varies between 380 and 520 sidereal days, $a^{R}$ is ranging from 0.0004 to $0.001 \mathrm{deg} / \mathrm{h}$ and $a^{I}$ varies 
1 between $-10^{-4}$ and $10^{-4} \mathrm{deg} / \mathrm{h}$. Therefore we have defined a priori locally uniform laws for 2 these parameters.

The pdfs obtained from the Bayesian estimation are plotted in Fig. 3. The mean values of the FCN parameters computed from the marginal probability laws are $T=428+/-3$ days, $a^{R}=(0.667+/-0.005) 10^{-3} \mathrm{deg} / \mathrm{h}$ and $a^{I}=(-0.492+/-0.05) 10^{-4} \mathrm{deg} / \mathrm{h}$. The law for $Q$ is not symmetric (and not Gaussian) so we define confidence intervals: $Q$ is between 7762 and 31989 within $90 \%$ C.I. Note the good agreement with the Levenberg-Marquardt least-squares results represented by the vertical dotted lines.

Based on the MHB Earth's parameter values and using Equation (3), this resonance strength estimate leads to an observed determination of the $\widetilde{\delta}_{1}$ internal pressure gravimetric factor of $\widetilde{\delta}_{1}=0.0306-i 0.00223$ with an error of $210^{-4}$. Theoretical values of the rotational pressure gravimetric factor $\widetilde{\delta}_{1}$ have been computed by Dehant et al. (1993). A first experimental determination of $\widetilde{\delta}_{1}$ has been proposed by Hinderer et al. (1991b) by stacking gravity measurements. They have found $\widetilde{\delta}_{1} \approx 0.043$ and they have estimated that the imaginary part of $\widetilde{\delta}_{1}$ is $1.6 \%$ of the real part, i.e. around $710^{-4}$. In our case $\widetilde{\delta}_{1}^{I}$ is about $7 \%$ of $\widetilde{\delta}_{1}^{R}$. From the FCN frequency, the combination of the dynamic flattening of the fluid core and the compliance (or the displacement Love number $h_{1}^{f}$ ) can be evaluated from $\widetilde{\sigma}_{n d}^{\prime}=-\Omega \frac{A}{A_{m}}\left(e^{f}-\beta\right)$ (cf. section 2). From our observed value, $\left(e^{f}-\beta\right) \approx 0.002$ which is in agreement with the MHB values of $e^{f}=2.645610^{-3}$ and $\beta=610^{-4}$ (Mathews et al., 2002).

\section{Conclusion}


The problem of negative $Q$-values encountered in previous studies has been avoided using the logarithm of $Q$ in the inversion scheme.

We have solved the FCN resonance damping equation for four parameters using on the one hand, the more traditional linearized least-squares method optimized with the LevenbergMarquardt algorithm, and on the other hand, a statistical Bayesian approach. We have demonstrated the good agreement between both results.

Because of the correlation existing between the quality factor $Q$ and the imaginary part of the resonance strength $a^{I}$, and the necessity for the mantle to possess some anelasticity, this latter parameter cannot be neglected and must be considered in the inversion scheme. Because of the large error on the phase of the diurnal waves close to the resonance, the parameter $x=$ $\log _{10}(Q)$ does not follow a Gaussian law.

From stacking the SG tidal gravity data, we can improve the determination of the FCN resonance parameters, which are in agreement with VLBI nutation determination. In order to further improve these estimates, the ocean loading correction must be improved at these diurnal frequencies. The nutation has the advantage to be less affected by the ocean loading uncertainty as the contribution of the ocean to the Earth's wobble is much smaller than its effect (mainly local) on the surface gravity.

Time-varying gravity data appear to provide complementary information to the VLBI nutation data as the latter cannot determine the precession while gravity can determine the $K_{1}$ tidal wave. Besides, gravity observations enable to retrieve additional Earth's interior parameters like the internal pressure Love numbers.

\section{Acknowledgements}

We acknowledge GGP members (http://www.eas.slu.edu/GGP/ggphome.html) for providing SG data. We thank also two anonymous referees for their comments on the manuscript. 


\section{$1 \quad$ References}

2 Amalvict, M., H. McQueen, and R. Govind, 2001. Absolute Gravity Measurements and

3 Calibration of SG-CT031 at Canberra, 1999-2000, J. Geodetic Soc. Jpn., 47(1), 410-416.

4

5 Crossley, D., Hinderer, J., Casula, G., Francis, O., Hsu, H.T., Imanishi, Y., Jentzsch, G.,

6 Kääriäinen, J., Merriam, J., Meurers, B., Neumeyer, J., Richter, B., Shibuya, K., Sato, T., Van

7 Dam, T., 1999. Network of superconducting gravimeters benefits a number of disciplines, 8 EOS, 80, 11, 121/125-126.

9

Geophys. Res., 98, 2091-2104. 
1 Dehant, V., 1987. Tidal parameters for an inelastic Earth. Physics of the Earth and Planetary

2 Interiors, 49, 97-116.

3

4 Dehant, V., Hinderer, J., Legros, H. and Lefftz, M., 1993. Analytical approach to the 5 computation of the Earth, the outer core and the inner core rotational motions, Phys. Earth 6 Planet. Int., 76, 259-282.

7

Ducarme, B., Sun, H.-P., Xu, J.-Q., 2007. Determination of the free core nutation period from tidal gravity observations of the GGP superconducting gravimeter network, J. Geod., 81, 179-187.

Ducarme, B., Rosat, S., Vandercoilden, L., Xu, J.Q., Sun, H.P., 2009. European tidal gravity observations: Comparison with Earth Tides models and estimation of the Free Core Nutation (FCN) parameters, Proceedings of the 2007 IAG General Assembly, Perugia, Italy, July 2 13, 2007, Observing our Changing Earth, M.G. Sideris (ed.), Springer Verlag, International Association of Geodesy Symposia 133, 523-532(DOI10.1007/978-3-540-85426-5).

Florsch, N., Chambat, F., Hinderer, J., \& Legros, H., 1994. A simple method to retrieve the complex eigenfrequency of the Earth's nearly diurnal free wobble; application to the Strasbourg superconducting gravimeter data, Geophys. J. Int., 116, 53-63.

Florsch, N. and Hinderer, J., 2000. Bayesian estimation of the free core nutation parameters from the analysis of precise tidal gravity data, Phys. Earth Planet Int., 117, 21-35.

Herring, T., Gwinn, C. \& Shapiro, I., 1986. Geodesy by radiointerferometry: studies of the 
1 forced nutations of the Earth, 1, Data analysis, J. Geophys. Res., 91, 4745-4755.

2

3 Hinderer, J., 1986. Resonance effects of the Earth's fluid core, in: A. Cazenave (Editor), Earth

4 Rotation: Solved and Unsolved Problems, Reidel, Dordrecht, 277-296.

5 Hinderer, J. And Legros, H., 1989. Elasto-gravitational deformation, relative changes in

6 gravity and earth dynamics, Geophys. J., 97, 481-495.

7

Hinderer, J., H. Legros \& D. Crossley, 1991a. Global Earth dynamics and induced gravity changes, J. Geophys. Res., 96, 20257-20265.

Hinderer, J., Zürn, W. \& Legros, H., 1991b. Interpretation of the strength of the nearly diurnal free wobble resonance from stacked gravity tide observations, in Proc. $11^{\text {th }}$ Int. Symp. Earth Tides, ed. J. Kakkuri, Schweitzerbart. Verlag, Stuttgart, 549-555.

Koot, L., Rivoldini, A., de Viron, O. and Dehant, V., 2008. Estimation of Earth interior parameters from a Bayesian inversion of very long baseline interferometry nutation time series, J. Geophys. Res., 113, B08414, doi:10.1029/2007JB005409.

Lambert, S.B. and Dehant, V., 2007. The Earth's core parameters as seen by the VLBI, Astr. \& Astr., 469, 777-781.

Legros, H., Hinderer, J., Lefftz, M. and Dehant, V., 1993. The influence of the solid inner core on gravity changes and spatial nutations induced by luni-solar tides and surface loading, Phys. Earth Planet. Int., 76, 283-315. 
2 Llubes, M., N. Florsch, J.P. Boy, M. Amalvict, P. Bonnefond, M.N. Bouin, S. Durand, M.F.

3 Esnoult, P. Exertier, J. Hinderer, M.F. Lalancette, F. Masson, L. Morel, J. Nicolas, M.

4 Vergnolle, G. Wöppelmann, 2008. A multi-technique monitoring of ocean loading in North of

5 France, C.R. Geoscience, 340, 379-389, doi:10.1016/j.crte.2008.03.005.

6

7 Lyard F., Lefevre F., Letellier T., Francis O., 2006. Modelling the global ocean tides: modern 8 insights from FES2004, Ocean Dynamics, 56, 394-415.

9

Mathews, P.M., Herring, T.A. and Buffet, B.A., 2002. Modeling of nutation and precession:

New nutation series for nonrigid Earth and insights into the Earth's interior, J. Geophys. Res., 107 (B4), 2068, 10.1029/2001JB000390.

Merriam, J. B., 1994. The nearly diurnal free wobble resonance in gravity measured at Cantley, Quebec, Geophys. J. Int, 119, 369-380.

Neuberg, J., Hinderer, J. \& Zürn, W., 1987. Stacking gravity tide observations in Central Europe for the retrieval of the complex eigenfrequency of the nearly diurnal free wobble, Geophys. J. R. Astr. Soc., 91, 853-868.

Press, W.H., Teukolsky, S.A., Vetterling, W.T., Flannery, B.P., 1992. Numerical Recipes in FORTRAN: the Art of Scientific Computing, second ed. Cambridge Univ. Press, Cambridge. 
2 Richter, B., \& Zürn, W., 1986. Chandler effects and the nearly diurnal free wobble as

3 determined from observations with a superconducting gravimeter, in The Earth's rotation and

4 reference frames for Geodesy and Geodynamics, eds. Babcock, A. \& Wilkins, G., Kluwer,

5 Dordrecht, 309-315.

6

7 Roosbeek, F., Defraigne, P., Feissel, M. and Dehant, V., 1999. The free core nutation period $8 \quad$ stays between 431 and 434 sidereal days, Geophys. Res. Lett., 26 (1), 131-134.

9

Rosat, S., and Lambert, S.B. 2009. FCN resonance parameters from VLBI and Superconducting Gravimeter data, submitted to Astronomy \& Astrophysics. 389. 
1 Tarantola, A., 2005. Inverse Problem Theory and Model Parameter Estimation, SIAM.

2

3 Tarantola, A., and Valette, B., 1982a. Inverse Problems = Quest for Information. J. Geophys., $4 \quad 50: 159-170$.

5

6 Tarantola, A., \& Valette, B., 1982b. Generalized nonlinear inverse problems solved using the 7 least squares criterion, Rev. Geophys. Space Phys., 20, 2, 219-232.

8

9 Vondràk, J. and Ron, C., 2006. Resonant period of free core nutation - its observed changes and excitations, Acta Geodyn. Geomater., vol. 3, No. 3 (143), 53-60.

Wahr, J.M., 1974. Body tides on anelliptical, rotating, elastic and oceanless Earth. Geophys. J. Astr. Soc., 64, $677-703$. tidal variations in rotation rate, Geophys. J. R. Astr. Soc., 87, 633-668. individual tidal records, in Proc. $11^{\text {th }}$ Int. Symp. Earth Tides, ed. J. Kakkuri, Schweitzerbart. 
2 Table captions

3 Table 1: A summary of various estimates of period and quality factor of the FCN. In addition

4 to theoretical results relative to an elastic Earth, to a slightly inelastic one and to MHB2000

5 model of Mathews et al. (2002), we have added experimental results from the IDA

6 (International Digital Accelerometers) network of spring gravimeters and from VLBI (Very

7 Long Baseline Interferometry). The other results are from superconducting gravimeter (SG)

8 datasets: $\mathrm{B}=$ Brussel (Belgium), $\mathrm{BH}=$ Bad Homburg (Germany), ST = Strasbourg (France),

$9 \mathrm{CA}=$ Cantley (Canada), $\mathrm{J}=3$ Japanese stations, $\mathrm{ES}=$ Esashi (Japan), MA $=$ Matsushiro

10 (Japan), $\mathrm{CB}=$ Canberra (Australia), $\mathrm{MB}=$ Membach (Belgium). 


\section{Figure captions}

2 Fig.1 Influence of the error of the imaginary part of the gravimetric factors on the resulting

3 pdfs for $a^{R}, a^{I}, x$ and $T$ retrieved from the Bayesian analysis of synthetic data. The error is expressed in percent of the gravimetric factor (imaginary part) amplitude. The relative error is (a) $10 \%$ for the 9 diurnal waves $Q_{1}, O_{1}, M_{1}, P_{1}, K_{1}, \Psi_{1}, \Phi_{1}, J_{1}$ and $O O_{1}$; (b) $10 \%$ for the 7

6 waves $Q_{1}, O_{1}, M_{1}, P_{1}, K_{1}, J_{1}, O O_{1}$ and $100 \%$ for $\Psi_{l}$ and $\Phi_{l}$; (c) $10 \%$ for $Q_{1}, O_{1}, M_{1}, P_{1}, K_{l}, J_{l}$,

$7 \quad O O_{1}$ and $200 \%$ for $\Psi_{l}$ and $\Phi_{l}$; (d) $50 \%$ for $Q_{1}, O_{l}, M_{l}, P_{l}, K_{l}, \Psi_{l}, \Phi_{l}, J_{l}$ and $O O_{l}$; (e) $100 \%$

8 for $Q_{1}, O_{1}, M_{1}, P_{1}, K_{1}, \Psi_{1}, \Phi_{1}, J_{1}$ and $O O_{1}$. The vertical dot lines indicate the values of $x, T, a^{R}$

9 and $a^{I}$ used to compute the synthetic delta-factors: $x=4, T=430$ days, $a^{R}=610^{-4} \mathrm{deg} / \mathrm{h}$ and $10 \quad a^{I}=-510^{-5} \mathrm{deg} / \mathrm{h}$.

Fig. 2 Joint and marginal pdfs for the FCN parameters $\left(a^{R}, T\right.$ and $\left.x\right)$ estimated from Strasbourg SG tidal gravity factors using the Bayesian method with (a) $a^{I}=0 \mathrm{deg} / \mathrm{h}$; (b) $a^{I}=$ $510^{-5} \mathrm{deg} / \mathrm{h}$. The Marquardt-least squares inversion results are also indicated as vertical dotted lines.

Fig. 3 Joint and marginal pdfs for the FCN parameters $\left(a^{R}, a^{I}, T\right.$ and $\left.x\right)$ estimated from stacking 7 European SG (Bad-Homburg, Moxa, Membach, Medicina, Strasbourg, Vienna, Wettzell) records using the Bayesian method. The Marquardt-least squares inversion results are also indicated as vertical dotted lines. 


\begin{tabular}{|c|c|c|c|}
\hline Author & Data & $\mathrm{T}$ & Q \\
\hline Neuberg et al. (1987) & Stacked Gravity $(\mathrm{B}+\mathrm{BH})$ & $431+/-6$ & $2800 \pm 500$ \\
\hline Sasao et al. (1980) & Theory elastic & 465 & $\propto$ \\
\hline Wahr \& Bergen (1986) & Theory anelastic & 474 & 78000 \\
\hline Herring et al. (1986) & VLBI & $435+/-1$ & $22000-10^{5}$ \\
\hline $\begin{array}{c}\text { Cummins \& Wahr } \\
\text { (1993) }\end{array}$ & Stacked Gravity IDA & $428+/-12$ & $3300-37000$ \\
\hline Sato et al. (1994) & Stacked Gravity J & $437+/-15$ & $3200-\propto$ \\
\hline Defraigne et al. (1994) & $\begin{array}{c}\text { Stacked Gravity } \\
\text { VLBI } \\
\text { Stacked Gravity + VLBI }\end{array}$ & $\begin{array}{c}424+/-14 \\
432+/-4 \\
433+/-3\end{array}$ & $\begin{array}{c}2300-8300 \\
Q>15000 \\
Q>17000\end{array}$ \\
\hline Florsch et al. (1994) & Gravity ST & $431+/-1$ & $1700-2500$ \\
\hline Merriam (1994) & Gravity CA & $430+/-4$ & $5500-10000$ \\
\hline Hinderer et al. (1995) & Stacked Gravity $(\mathrm{ST}+\mathrm{CA})$ & $429+/-8$ & $7700-\propto$ \\
\hline Roosbeek et al. (1999) & VLBI & $431-434$ & - \\
\hline $\begin{array}{l}\text { Florsch and Hinderer } \\
\qquad(2000)\end{array}$ & Gravity ST (Bayes) & 428 & $Q>20000$ \\
\hline Hinderer et al. (2000) & Gravity + VLBI & $431-434$ & $15000-30000$ \\
\hline Mathews et al. (2002) & MHB2000 model & $\begin{array}{l}430.20+/- \\
0.28\end{array}$ & 20000 \\
\hline Sato et al. (2004) & Stacked gravity $(\mathrm{ES}+\mathrm{MA}+\mathrm{CB}+\mathrm{MB})$ & $429.7+/-1.4$ & $9350-10835$ \\
\hline Vondrák \& Ron (2006) & VLBI & $\begin{array}{l}430.32+/- \\
0.07\end{array}$ & $20600+/-340$ \\
\hline Ducarme et al. (2007) & Mean gravity & $429.7+/-2.4$ & Not estimated \\
\hline $\begin{array}{l}\text { Lambert \& Dehant } \\
\text { (2007) }\end{array}$ & VLBI & $430+/-0.4$ & $17000+/-3000$ \\
\hline Ducarme et al. (2009) & Mean gravity in Europe & $430+/-2$ & $15000+/-8000$ \\
\hline Koot et al. (2008) & VLBI (Bayes) & 430 & $13750+/-514$ \\
\hline This paper & $\begin{array}{c}\text { Stacked gravity of } 7 \text { European SGs } \\
\text { (Bayes) }\end{array}$ & $428+/-3$ & $7762<\mathrm{Q}<31989$ (90\% C.I. $)$ \\
\hline
\end{tabular}

Table 1 
(a) $10 \%$ on $\delta^{\mathrm{I}}$ for the 9 diurnal waves $Q_{1}, O_{1}, M_{1}, P_{1}, K_{l}, \Psi_{1}, \Phi_{l}, J_{l}$ and $O O_{1}$
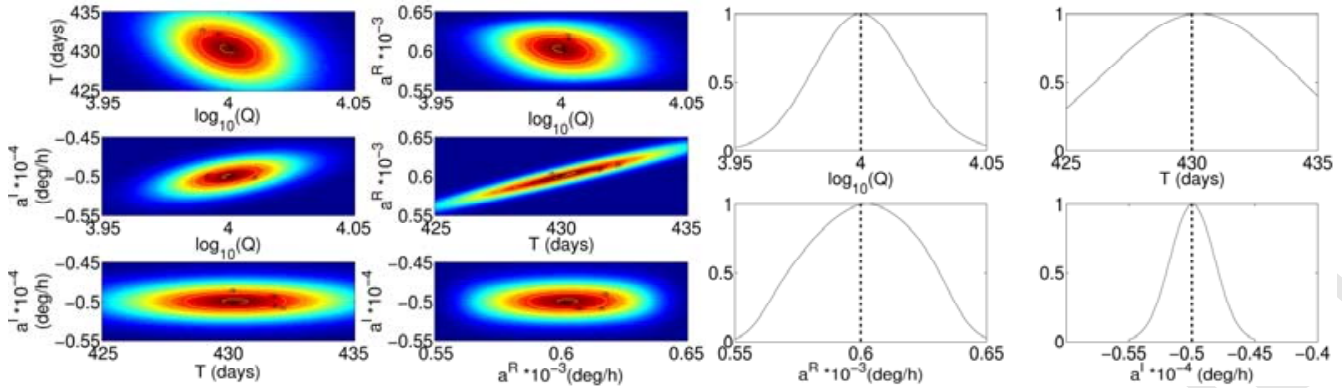

(b) $10 \%$ on $\delta^{\mathrm{I}}$ for the 7 main waves and $100 \%$ on $\delta^{\mathrm{I}}\left(\Psi_{1}\right)$ and $\delta^{\mathrm{I}}\left(\Phi_{1}\right)$
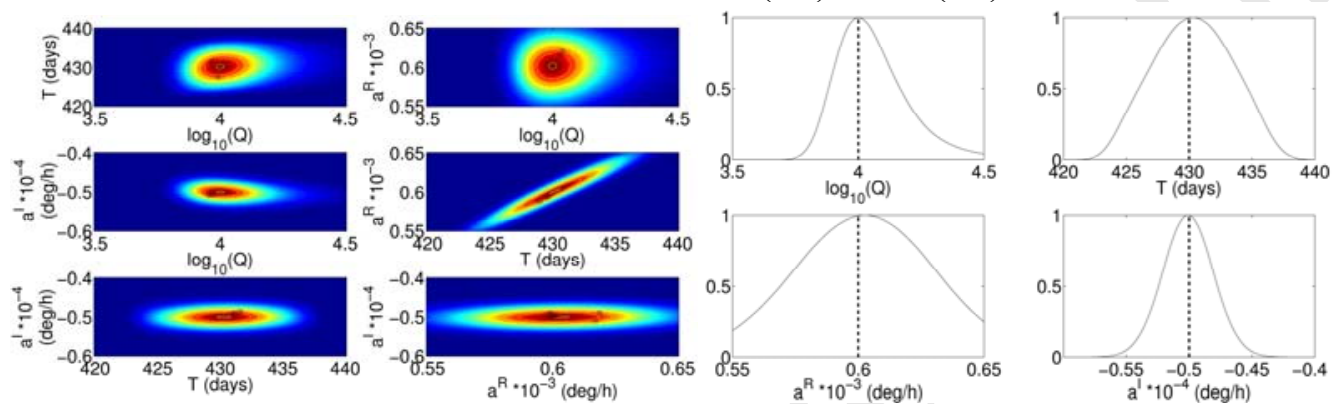

(c) $10 \%$ on $\delta^{\mathrm{I}}$ for the 7 main waves and $200 \%$ on $\delta^{\mathrm{I}}\left(\Psi_{1}\right)$ and $\delta^{\mathrm{I}}\left(\Phi_{1}\right)$
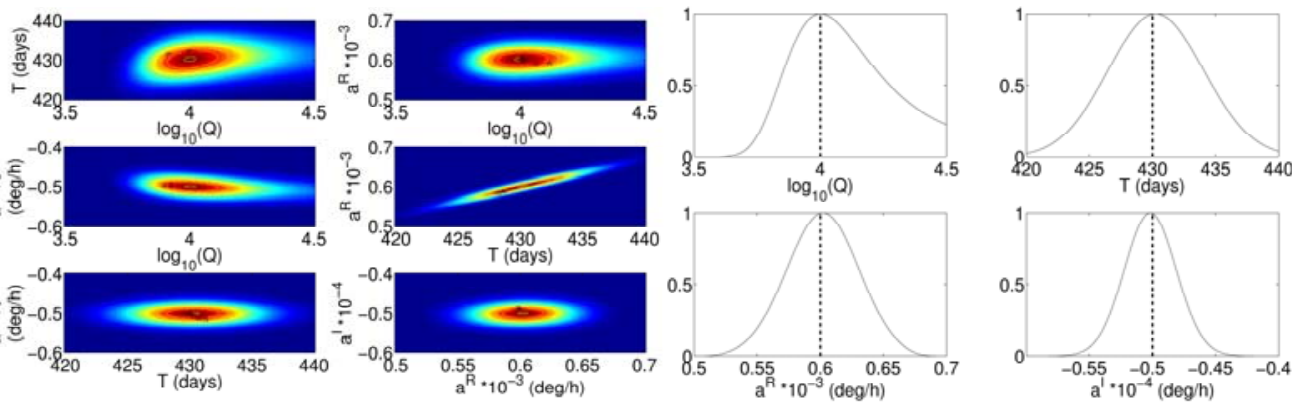

(d) $50 \%$ on $\delta^{\mathrm{I}}$ for the 9 diurnal waves $Q_{1}, O_{1}, M_{1}, P_{1}, K_{1}, \Psi_{1}, \Phi_{1}, J_{1}$ and $O O_{1}$
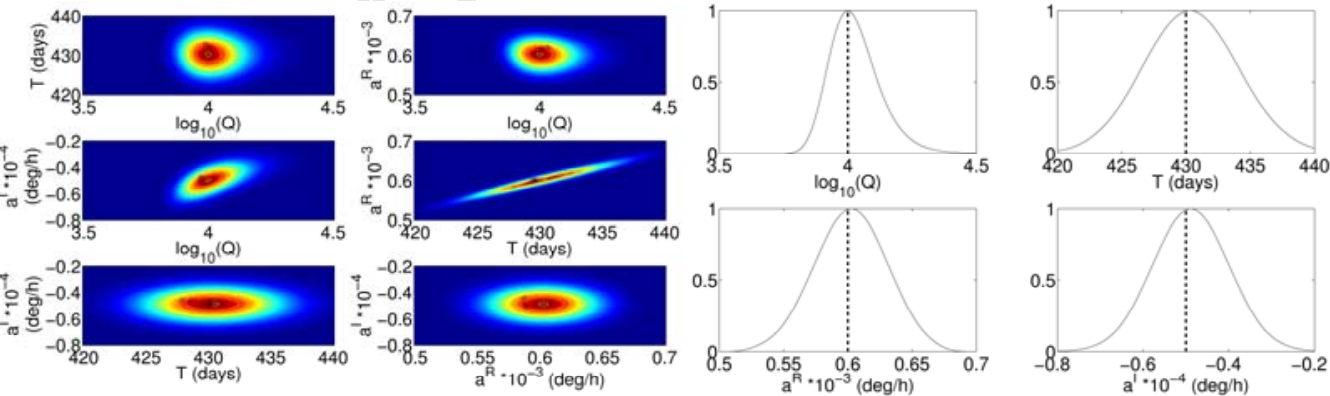

(e) $100 \%$ on $\delta^{\mathrm{I}}$ for the 9 diurnal waves $Q_{1}, O_{1}, M_{1}, P_{1}, K_{1}, \Psi_{1}, \Phi_{1}, J_{1}$ and $O O_{1}$
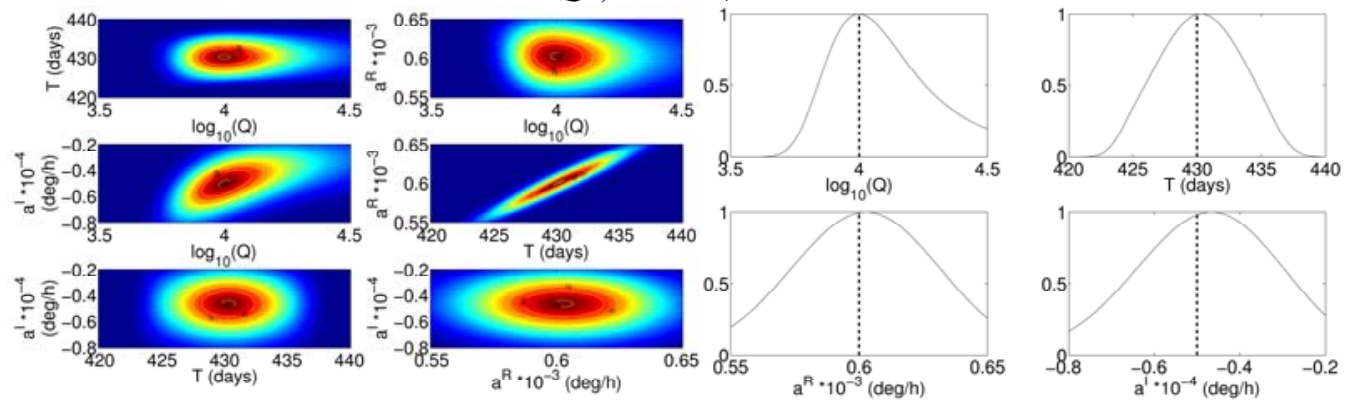

Fig. 1 
(a)

Strasbourg -3 parameters $-a^{I}=0 \mathrm{deg} / \mathrm{h}$
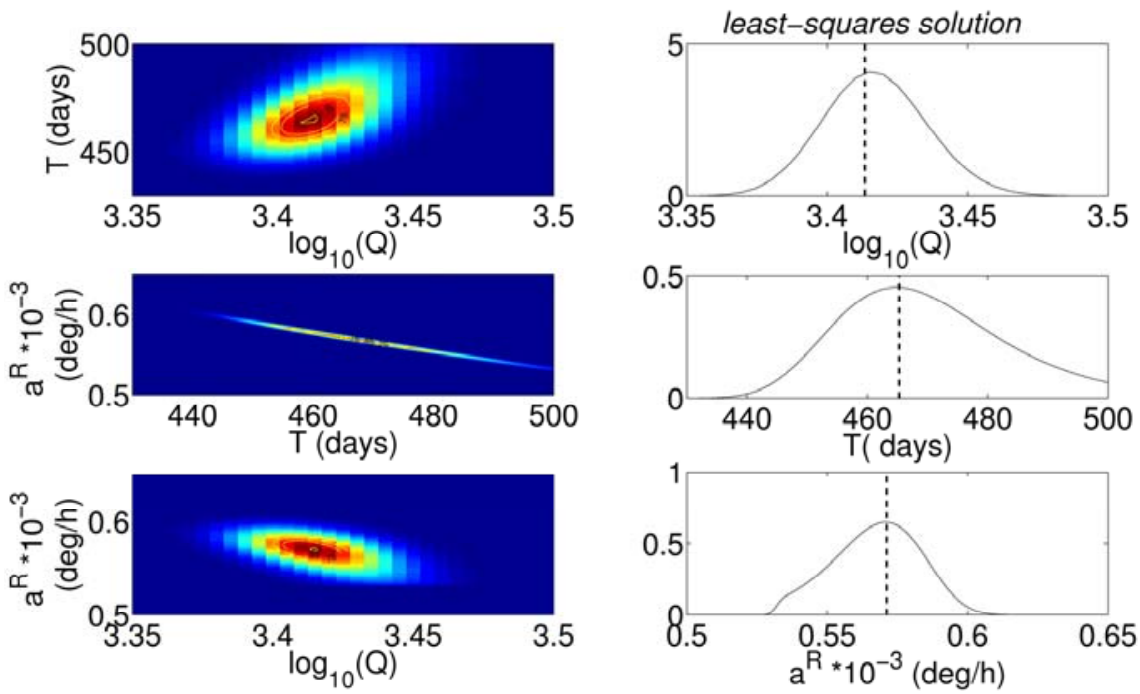

(b)

Strasbourg -3 parameters $-a^{I}=-510^{-5} \mathrm{deg} / \mathrm{h}$
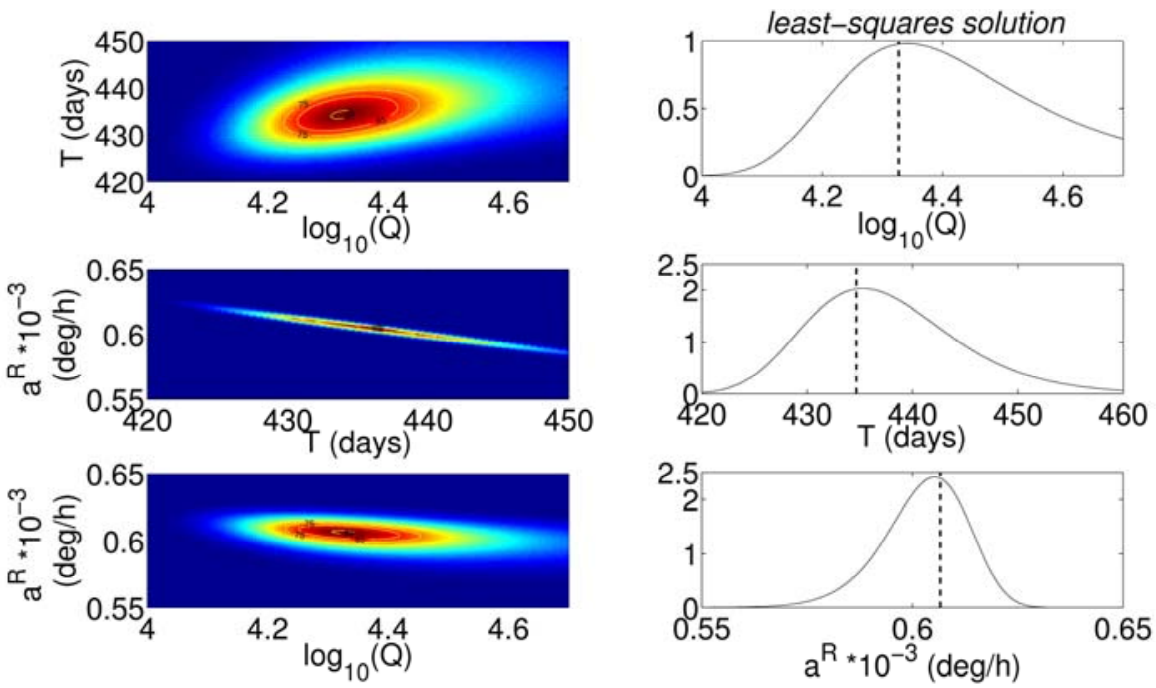

Fig. 2 
Stack of 7 European GGP-time series

$$
\text { (H1, M1, MB, MC, ST, VI, W1) }
$$
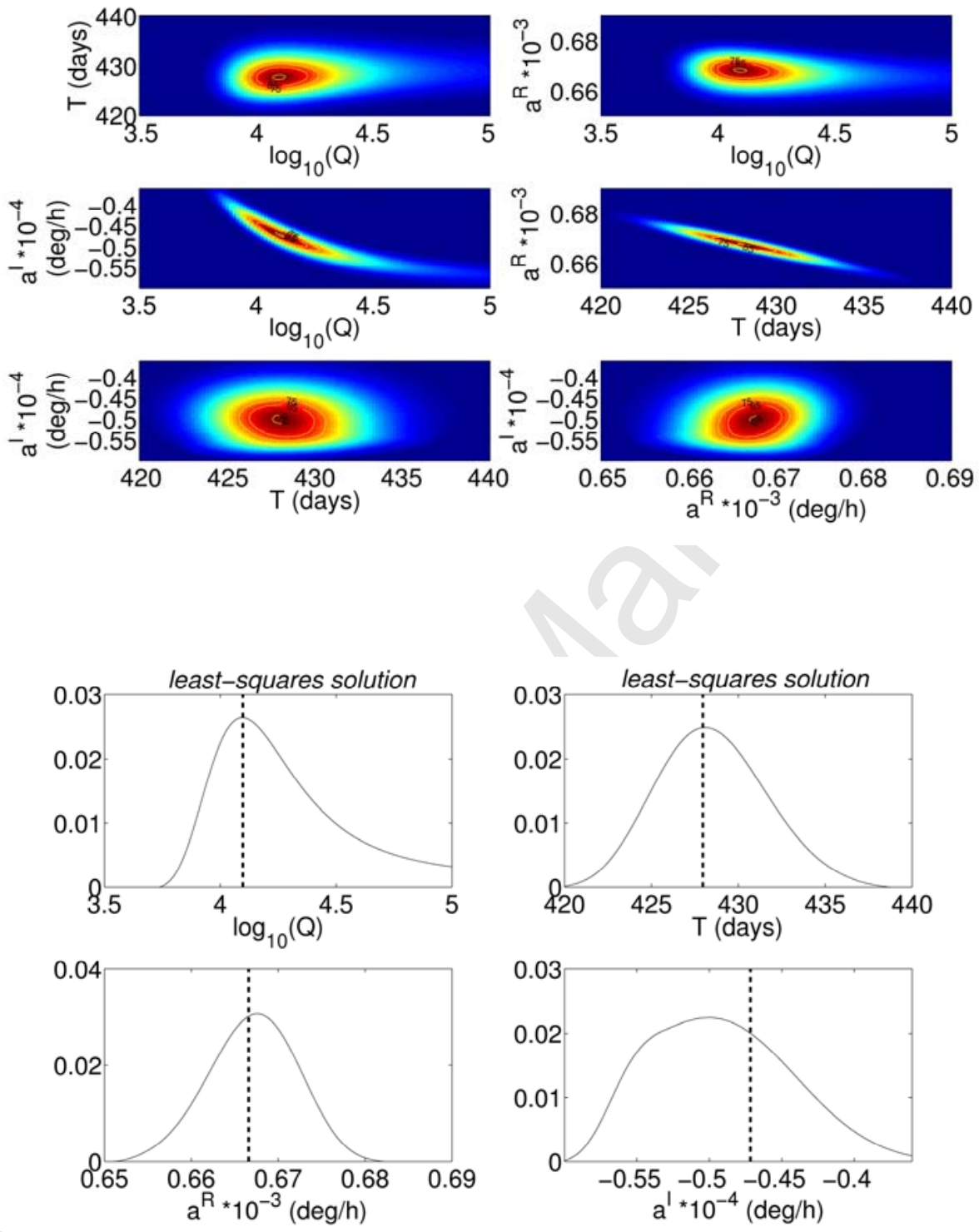

Fig. 3 